\title{
Distribution of practice effects on imprinting'
}

\author{
GLORIA J. FISCHER \\ WASHINGTON STATE UNIVERSITY
}

In support of a basic similarity, rather than difference, between imprinting and associative learning imprinting in chicks was found to be facilitated by distribution of practice. Specifically, as compared to a continuous 12-min. session, two 6-min. imprinting sessions with 10 or 30 -min. inter-sessionintervals reduced subsequent generalization of following from the parent to a strange model.

A well supported finding in infra-human and human learning is that it is facilitated by distribution of practice or rest periods between trials. The absence of such a finding in imprinting has been interpreted to support a distinction between imprinting and ordinary associative learning (Hess, 1957, 1964). Such an interpretation might be questioned, however. Data on which it was based (Hess, 1957), for example, were restricted to single trial (massed) sessions of variable duration, and total exposure time was not constant. A subsequent study (Sluckin \& Salzen, 1961) did distribute imprinting trials and held total exposure constant, by giving chicks either three 10-min. imprinting sessions on their first day of life or five 6-min. sessions on each of their first five days. The incidence of following under the two conditions still did not suggest facilitation from greater distribution. However, the daily 6-min. sessions extended practice beyond the critical period for imprinting, using socially reared chicks. Further, chicks were not retested to ascertain the extent of actual preference for the parent model. The validity of imprinting per se under such circumstances has been questioned (Sluckin \& Salzen, 1961; Polt \& Hess, 1964).

In an attempt to clarify the influence of distribution of practice on imprinting (and resulting implications for a distinction between imprinting and ordinary associative learning) the present study varied duration of rest period midway through the imprinting experience, while holding constant both total exposure time and distance traversed by the model. Imprinting was assessed both by the amount of following during the imprinting experience and by differential following of the parental model (as compared to a different one) on retest.

Method

When fluffy dry, ( 4 to $6 \mathrm{hr}$.), day-old broiler chicks were isolated in a darkened incubator room until imprinting at $18-1 / 2 \mathrm{hr}$. The first 80 able to stand and not crippled at imprinting or retesting were used in the experiment.

Description of the apparatus and imprinting procedure appear elsewhere (Fischer, 1966). Briefly, a suspended model traversed a circular runway in 6-1/2 sec. runs and 13-1/2 sec. pauses. The model was an
8 in. red cube, heated and emitting $12 \mathrm{sec}$. of repetitive tapping at the rate of two taps per sec., alternated with 6 sec. of silence. Both the red cube and a different model were used for retests. The different model was an orange, double truncated cone of comparable size. Its auditory stimulus consisted of continuous pleasure calls and peeps. Over-all SPL of noise (noise plus signal) measured $6 \mathrm{in}$. from the output source, was set at $65 \mathrm{db}$ for both tap and pleasure call tapes.

After $5 \mathrm{~min}$. adaptation in the runway to light, sound and model proximity, each chick was imprinted to the red cube and tap sound (RT). Twenty were assigned randomly to each of four groups whose imprinting schedule consisted of intermittent movement of RT through four runway revolutions (12 min.); with either $0,1,10$, or 30 min. between the second and third revolutions. The 0 min. (continuous, or massed practice) group was undisturbed during imprinting. For the other (two sessions, or distributed practice) groups light and sound were cut off at the end of the second revolution, and the chick was returned in the dark to the incubator room for one of the indicated inter-session-intervals. Seconds following within $1 \mathrm{ft}$. of the model was recorded during the first half (revolutions 1 and 2) and during the second half (revolutions 3 and 4) of imprinting. At $36 \mathrm{hr} .(18 \mathrm{hr}$. after imprinting) all chicks were retested to RT for four rounds, then $1 / 2 \mathrm{hr}$. later, to OP for four rounds. Seconds following within $1 \mathrm{ft}$. of each model was recorded for all four rounds during retests.

\section{Results and Discussion}

Data were analyzed for possible distribution of practice effects on both following during imprinting and imprinting strength, measured by differential following of the parent (RT) and different (OP) models during retests. Analysis of variance in seconds following during imprinting revealed a substantial increase from the first to the second half of the imprinting experience $(F=34.84$; $\mathrm{df}=1 / 76$ ). But this increase, shown in Table 1 , was unaffected by distribution of practice $(F<1.00$ for both distribution and for the interaction of distribution with trial halves). Thus, in agreement with prior findings, chicks appear to follow as well with one continuous imprinting experience as they do when the experience is distributed over two sessions.

Cell variances in following on retests were found to be heterogeneous $\left(\chi^{2}=26.14\right)$, but adequately reduced by

Table 1. Mean Time (Sec) Following During Imprinting
First Half 83.2
Second Half 140.4 


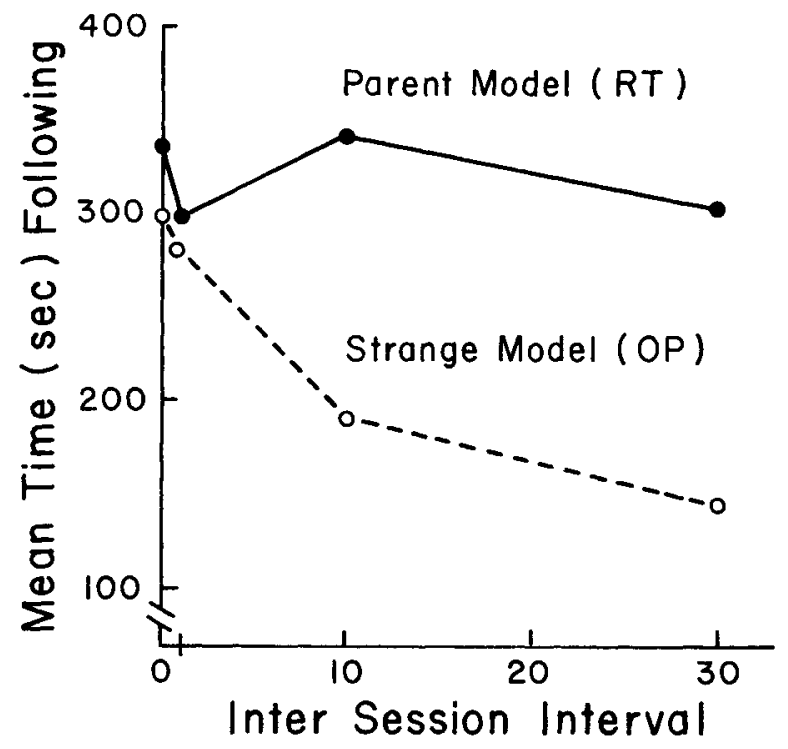

Fig. 1. Time (sec.) following parent and strange models on retest, after imprinting with massed $(0)$, interrupted (1) or inter session (10 or 30 min.) intervals.

a $\sqrt{x}+\sqrt{x+1}$ transformation $\left(x^{2}=5.97\right)$. Analysis of variance then in transformed following scores revealed substantial effects from distribution of practice. The nature of the effect is shown in Fig. 1. Since it took $1 \mathrm{~min}$. to discontinue light and sound and to transport chicks from the apparatus to the incubator room and back, the $1 \mathrm{~min}$. inter-session-interval (ISI) group constitutes a control for the effects of disturbance and handling. Generalization to a strange model was very considerable for this group, but no greater than for the undisturbed or 0 ISI group ( $F<1.00$ for differences between models at 0 and $1 \mathrm{~min}$. ISI). With intervals as long as 10 or $30 \mathrm{~min}$. midway through imprinting, however, generalization to a strange model was significantly reduced. (For differences between $R T$ and $O P$ at 10-min. and $30-\mathrm{min}$. ISI, $\mathrm{F}=4.58$ and $\mathrm{F}=5.43$, respectively; $\mathrm{df}=1 / 76$. )

Present findings then clearly support a facilitating effect on imprinting from distribution of practice. Speci- fically, two 6-min. imprinting sessions separated by 10 or $30 \mathrm{~min}$. intervals reduced the extreme generalization from parent to another model found with one 12min. imprinting session. Findings point out too, the probable reason why prior studies failed to find a distribution effect, It was not found to increase following the imprinting model because it does not in fact do so, either during imprinting or on retest. Rather, distribution reduces subsequent generalization of following, i.e., increases discriminability between parent and strange models. One implication is that still another apparent difference between imprinting and ordinary associative learning is thereby eliminated. Another, (see also Polt \& Hess, 1964), is that imprinting can not be assessed merely by the tendency to follow during an imprinting experience. This response might better be called an "approach fixation" (Schneirla, 1965), with the term imprinting reserved for approach fixations selectively evoked by the imprinting stimulus. So defined, imprinting may be more sensitively measured by differential following on retest than by the choice-ofmodel method (see Klopfer, 1965).

\section{References}

Fischer, Gloria J. Auditory stimuli in imprinting. J. comp. physiol. Psychol., 1966, 61, 271-273.

Hess, E. H. Effects of meprobamate on imprinting in waterfowl. Ann. N. Y. Acad. Sci., 1957, 67, 724-732.

Hess, E. H. Imprinting in birds. Science, 1964, 146, 1128-1139.

Klopfer, P. H. Imprinting: a reassessment. Science, 1965, 147 , 302-303.

Polt, J. M., \& Hess, E. H. Following and imprinting: effects of light and social experience. Science, 1964, 143, 1185-1187.

Schneirla, T. C. Aspects of stimulation and organization in approach/withdrawal processes underlying vertebrate behavioral development. In D. L. Lehrman, R. Hinde, \& Evelyn Shaw (Eds.), Advances in the study of behavior. New York: Academic Press, 1965. Pp. 1-74.

Sluckin, W., \& Salzen, E. A. Imprinting and perceptual learning. Qtr. J. exp. Psychol., 1961, 13, 65-77.

\section{Note}

1. This study was supported by Research Grant MH 08438-02 from the National Institute of Mental Health, United States Public Health Service. Sara C. Gilman and Jerome Uhlman assisted with data collection. Results were first presented at the 1966 meetings of the Midwestern Psychological Association. 